\title{
50 Years Niels Bohr Institute, Copenhagen
}

The following appreciation is based on the booklet "The Niels Bohr Institute. October 7, 1965" and on additional information kindly supplied to Europhysics News by Ove Nathan.

7 October 1965 marked the eightieth anniversary of Niels Bohr's birth. On that day the Institute for Theoretical Physics of the University of Copenhagen was given the name it had already carried unofficially for many years: The Niels Bohr Institute. Having been officially opened in 1921, the Institute this year celebrates its fiftieth anniversary. It occupies a unique place in the history of modern atomic theory and of nuclear physics. To present here a brief review of its development is therefore amply justified.

Niels Bohr's first epoch-making papers on the quantum theory of atomic structure were published in 1913 when he was assistant professor at the University of Copenhagen. A year later he went to Manchester as a reader in mathematical physics upon invitation by Rutherford, and in 1916 he was appointed professor of a new chair in theoretical physics at Copenhagen. In the beginning he was assisted by a secretary and a mechanic and had only a small office on Sølvgade; his first scientific assistant was the young Dutch theoretical physicist H.A. Kramers. Soon Bohr took the initiative of proposing the creation of a separate institute. Ground was purchased on Blegdamsvej with funds collected by a group of persons ; the building was donated by the Danish government. The Carlsberg Foundation (the Carlsberg Company is a famous brewery) provided annual grants in support of the scientific investigations. In 1921 the Institute was officially dedicated and Niels Bohr became its first director, a position he held until his death in 1962 ; then his son Aage took over the directorship.

The early 1920's saw the dawn of a new and glorious period in physics, that of quantum mechanics. Discoveries followed one another, completely revolutionizing atomic theory, and young physicists flocked to centres

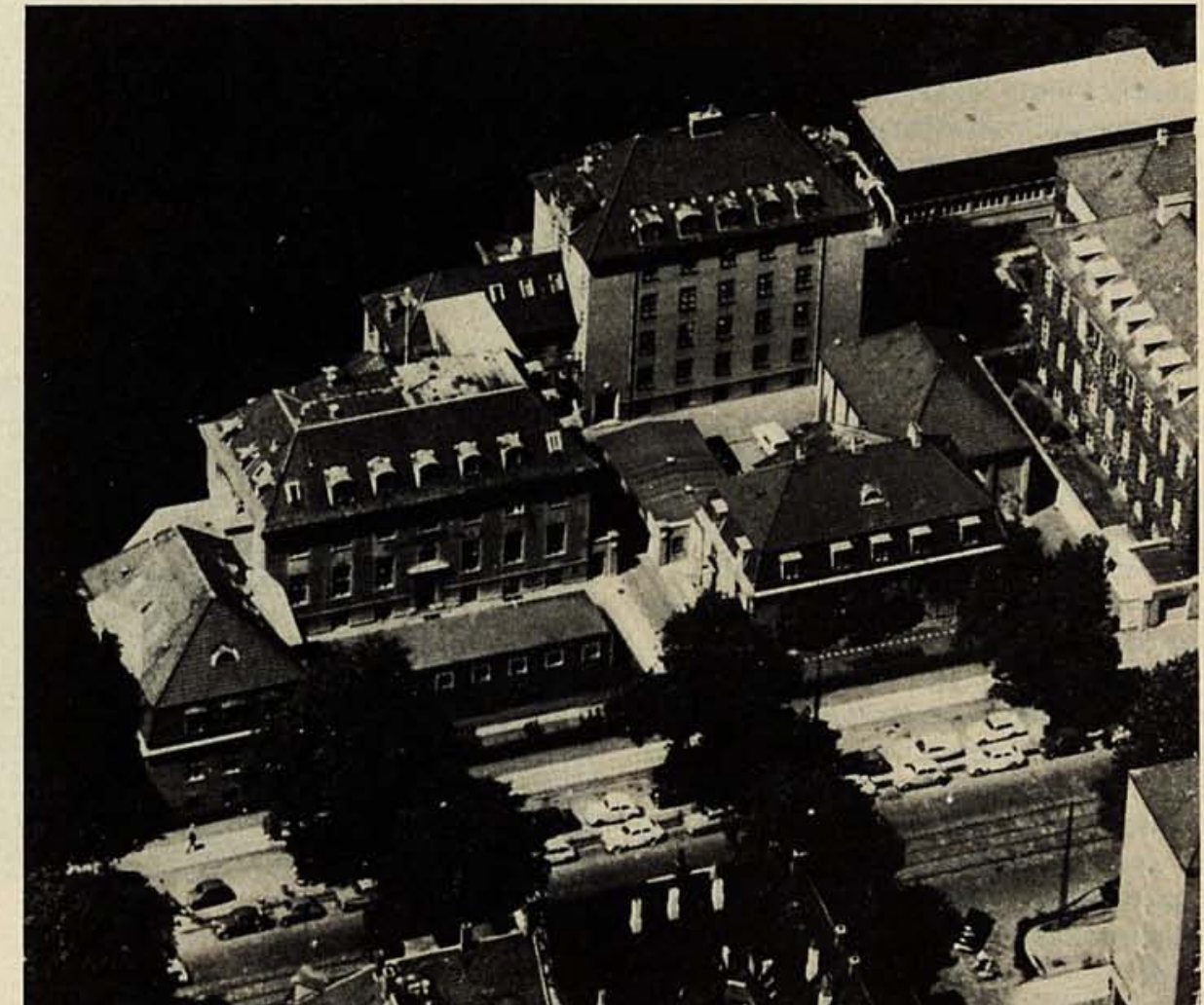

at Copenhagen, Göttingen and Cambridge to participate in its development. It was a time when the Göttingen mathematician David Hilbert remarked that Nobel Prizes were lying in the street.

In spite of its name, there was a considerable amount of experimental physics carried out at the Copenhagen Institute, concentrating on spectroscopic investigations to test and stimulate the development of the new theory. For example, Coster and Hevesy discovered a new element with just the properties predicted by Bohr's theory, by means of X-ray spectra taken at the Institute. It was called hafnium after Hafnia (Copenhagen).

The Institute soon became too small to house the enthusiastic young crowd from all over the world. The means for its extension, realized between 1924 and 1926, were made available by the International Education Board (later the Rockefeller Foundation) in the USA, and the city of Copenhagen donated the additional land.

The latter part of the 1920's saw a great many scientists at the Institute discussing with Bohr and his collaborators in particular the epistemological aspects of Heisenberg's uncertainty relations and Bohr's concept of "complementarity" in quantum physics and other domains of our experience. Heisenberg, Pauli, Klein, Dirac and Landau, to mention just a few, were among the prominent visitors at Copenhagen.

After this first exciting period emphasis shifted gradually from atomic physics to the properties of nuclei and elementary particles. At an early stage of this development the Copenhagen Institute was able to participate in this research thanks to the installation of new laboratories with a high-voltage generator and a cyclotron. Funds for these facilities were made available by the Carlsberg and Thrige Foundations, as well as by the Rockefeller Foundation. 
In those years radium-beryllium sources were used to produce neutrons. On his fiftieth birthday (1935) a group of Danish companies and foundations presented Niels Bohr with funds for the purchase of a strong radium source. The first interpretation of uranium fission was given by Frisch and Meitner on the basis of Bohr's liquid drop model of the nucleus (1939). Bohr also recognized the different roles of the two uranium isotopes in the fission process, a fact of great importance for the technical development towards atomic energy. From this period stems also the classical paper by Bohr and Wheeler on the liquid drop model for the nucleus.

The Copenhagen accelerator was also used to produce radioactive isotopes for biological research. Niels Bohr had inherited a deep interest in biological problems from his father; he enthusiastically supported the biological tracer research at his Institute, which research between 1934 and 1943 was directed by Hevesy.

The political development in Europe during the 1930's influenced the life at the Institute. Many refugees came to Copenhagen; some stayed and others continued with Bohr's help to England and the United States. Bohr himself left Denmark for Sweden in 1943 to avoid political persecution. He returned to Copenhagen in August 1945 and immediately planned an extension of the Institute to permit more active participation in nuclear research. From then on, highenergy physics became one of the main fields of research there. When the European Organization for $\mathrm{Nu}$ clear Research (CERN) was established in Geneva in 1953, the Institute was host to the CERN theoretical study group for several years.

In the early fifties Aage Bohr and Mottelson began the development of what is now known as the model of collective motions for the nucleus (" collective model" for short).

In 1956 the five Scandinavian countries (Denmark, Finland, Iceland, Norway and Sweden) agreed to establish together the Nordic Institute of

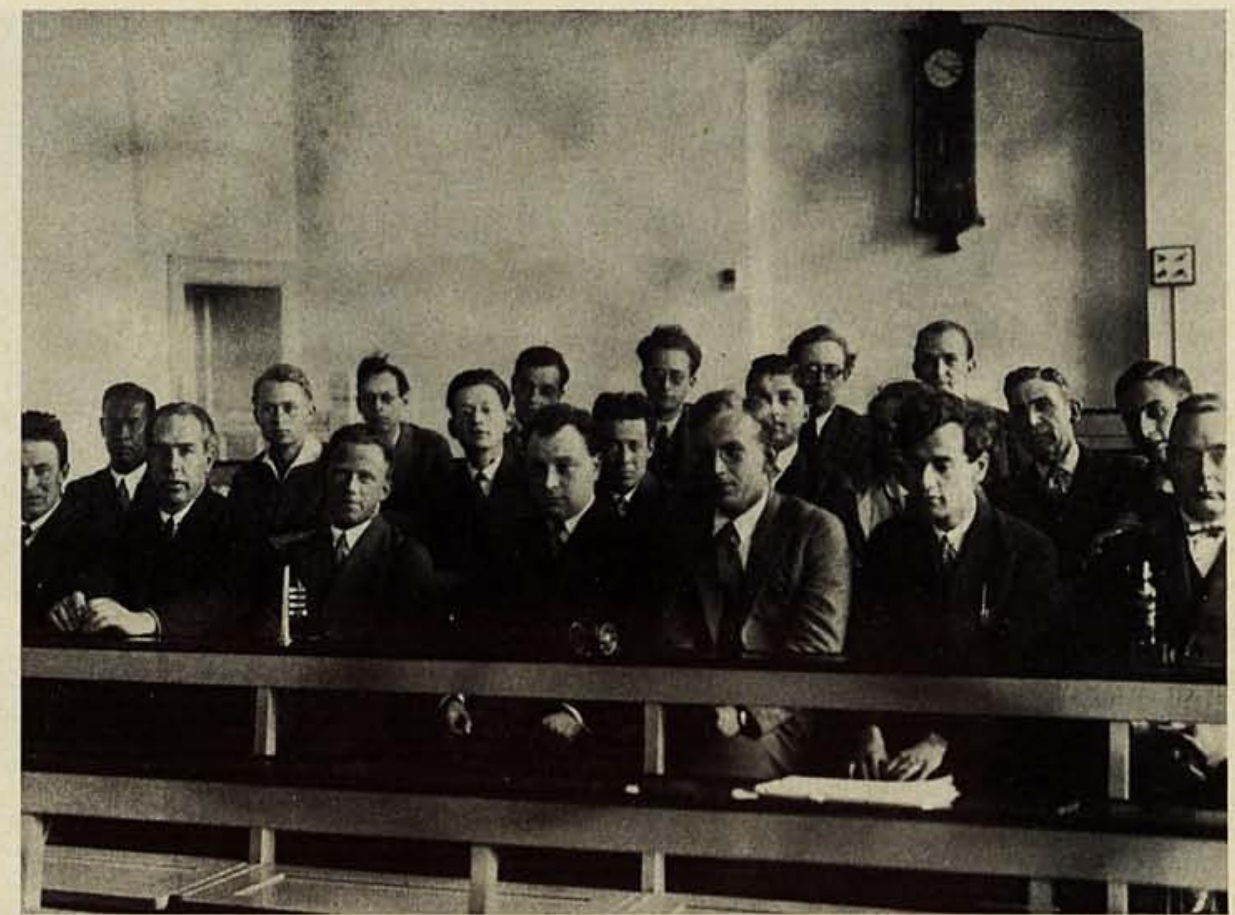

Theoretical Atomic Physics (NORDITA). This organization is located at the Niels Bohr Institute on Blegdamsvej; it offers scholarships to young Scandinavian physicists for participation in the scientific work at the Institute.

More recently the activities of the Niels Bohr Institute in physics research and advanced teaching in physics have broadened considerably. A large electrostatic generator of the tandem type was acquired in 1959 and placed on a site close to that of the Atomic Energy Establishment at Ris $\varnothing$, about $30 \mathrm{~km}$ west of Copenhagen. The most important development since 1965, when the Institute became officially the Niels Bohr Institute, has been the inauguration in September 1970 of a new tandem generator (of the super FN-type) which produces $18-19 \mathrm{MeV}$ protons. The work of the theoretical physics group, although covering a broad section of modern physics, has retained its emphasis on nuclear and highenergy physics.

At present, the Niels Bohr Institute has a staff of about 75 scientists with some 60 visitors from abroad each year, in addition to the about 25 physicists working with NORDITA. Over the years close to 1000 scientists have been associated with the Institute for short or long periods. To illustrate its scientific productivity, it should be noted that in 1969 more than 60 research papers were published, and about the same number in 1970.

The Niels Bohr Institute with its illustrious past stands out as a prime example of stimulating collaboration between physicists from many countries, reaching across all geographical and political barriers. It is confidently expected that it will also be fully capable of meeting the challenge of the future. 